\title{
DTI Analysis of Presbycusis Using Voxel-Based Analysis
}

\author{
(D)W. Ma, (D) M. Li, (D)F. Gao, (D)X. Zhang, (D). Shi, (D). Yu, (D)B. Zhao, (DW. Chen, (D) G. Wang, and (D). Wang
}

\begin{abstract}
BACKGROUND AND PURPOSE: Presbycusis is the most common sensory deficit in the aging population. A recent study reported using a DTI-based tractography technique to identify a lack of integrity in a portion of the auditory pathway in patients with presbycusis. The aim of our study was to investigate the white matter pathology of patients with presbycusis by using a voxel-based analysis that is highly sensitive to local intensity changes in DTI data.
\end{abstract}

MATERIALS AND METHODS: Fifteen patients with presbycusis and 14 age- and sex-matched healthy controls were scanned on a 3T scanner. Fractional anisotropy, mean diffusivity, axial diffusivity, and radial diffusivity were obtained from the DTI data. Intergroup statistics were implemented on these measurements, which were transformed to Montreal Neurological Institute coordinates by using a nonrigid image registration method called large deformation diffeomorphic metric mapping.

RESULTS: Increased axial diffusivity, radial diffusivity, and mean diffusivity and decreased fractional anisotropy were found near the right-side hearing-related areas in patients with presbycusis. Increased radial diffusivity and mean diffusivity were also found near a language-related area (Broca area) in patients with presbycusis.

CONCLUSIONS: Our findings could be important for exploring reliable imaging evidence of presbycusis and could complement an ROI-based approach.

ABBREVIATIONS: $\mathrm{DA}=$ axial diffusivity; $\mathrm{dB} \mathrm{HL}=$ decibel hearing level; $\mathrm{DR}=$ radial diffusivity $\mathrm{FA}=$ fractional anisotropy; $\mathrm{MD}=$ mean diffusivity; $\mathrm{PTA}=$ pure-tone average

A ge-related hearing loss, also known as presbycusis, is the most common sensory deficit in the aging population. Presbycusis is usually characterized by progressive hearing loss at high frequencies, which are particularly important for speech recogni-

Received January 28, 2016; accepted after revision May 9.

From the Department of Otolaryngology (W.M., L.S.), the Second Hospital of Shandong University, The Central Hospital of Jinan City (W.M., L.Y.), and Shandong Medical Imaging Research Institute (F.G., X.Z., B.Z., G.W.), Shandong University, Jinan, China; College of Electronics and Information Engineering (M.L.), Sichuan University, Chengdu, China; Philips Healthcare (W.C.), Shanghai, China; and the Department of Radiation Oncology (X.W.), University of Nebraska Medical Center, Omaha, Nebraska.

W. Ma and M. Li contributed equally to this work.

This project was supported by the National Natural Science Foundation of China (grants 81171380/H1807 and 81371534), Shandong Provincial Natural Science Foundation of China (grant BS2015YY003), and Shandong Provincial Medical and Healthy Technology Development Program of China (grant 2015WS0176).

Please address correspondence to Guangbin Wang, MD, Shandong Medical Imaging Research Institute, Jing-wu Rd No. 324, Jinan 250021, People's Republic of China; e-mail:wgb7932596@hotmail.com

-- Indicates open access to non-subscribers at www.ajnr.org

三 Indicates article with supplemental on-line table.

http://dx.doi.org/10.3174/ajnr.A4870 tion. Hypofunction of the inner ear is the main reason for the peripheral component of presbycusis. ${ }^{1}$ However, poor speech discrimination and deteriorated temporal sound processing reflect a possible central component of presbycusis. ${ }^{2,3}$ Moreover, many animal studies have showed the existence of a central component of presbycusis. ${ }^{4,5}$

Recent studies of multiple MR imaging modalities have demonstrated their capabilities of offering reliable imaging markers for recognizing presbycusis. In a structural MR imaging study, the volume and surface area were decreased in the auditory cortex areas of patients with presbycusis compared with young healthy controls. ${ }^{6}$ In a functional MR imaging study, patients with presbycusis showed higher blood oxygen level-dependent activation in response to acoustic stimuli in the temporal lobes compared with young healthy controls. ${ }^{7}$

The aforementioned imaging markers were mainly explored in gray matter, whereas white matter integrity, as one of the most sensitive indicators of axon damage or demyelination, requires further study. A widely used technique, DTI, has been considered as the most effective method for characterizing white matter organization. Multiple scalar measurements, including fractional 
Demographic and auditory function of the presbycusis and control groups

\begin{tabular}{lccc}
\hline \multicolumn{1}{c}{ Demographics } & Presbycusis & Control & $P$ Value \\
\hline No. of patients & 15 & 14 & \\
Age $(\mathrm{yr})^{\mathrm{a}}$ & $63.2 \pm 2.6$ & $62.4 \pm 2.0$ & $.38^{\mathrm{b}}$ \\
Female, no. (\%) & $10(66.7)$ & $8(57.1)$ & $.60^{\mathrm{c}}$ \\
Education levels $(\mathrm{yr})^{\mathrm{a}}$ & $10.7 \pm 3.5$ & $10.1 \pm 2.6$ & $.61^{\mathrm{b}}$ \\
PTA (dB HL) & $30.7 \pm 4.7$ & $15.9 \pm 1.4$ & $<.001^{\mathrm{b}}$ \\
\hline
\end{tabular}

a Data are given as mean \pm standard deviation.

b The between-group differences were assessed with the 2-tailed Student $t$ test

c The between-group differences were assessed with the 2-tailed Pearson $\chi^{2}$ test.

${ }^{\mathrm{d}}$ The PTAs from both ears were averaged.

anisotropy (FA), mean diffusivity (MD), axial diffusivity (DA), and radial diffusivity (DR), can be calculated from DTI data through tensor calculation. These measurements are useful indicators for characterizing various types of pathology in the human brain.

Many DTI studies have reported changes in the auditory pathway and auditory cortex in patients with sensorineural hearing loss. ${ }^{8,9}$ To our knowledge, only 1 study has reported a lack of white matter integrity in patients with mild presbycusis by applying an intergroup statistic on the ROI-based features, which was in the form of reconstructed auditory tracts. ${ }^{6}$ Besides that, the study showed no obvious differences in any measured DTI parameters between mild presbycusis and expressed presbycusis. Although promising, a more reliable imaging marker of presbycusis could be established by designing more sophisticated experiments. First, the reconstructed auditory pathway was partly obscured by the optic radiation. This so-called "fiber-crossing issue" could be mitigated by using probabilistic tractography; however, the "gold standard" for validating the tractography, especially in the fiber-crossing area, has not been established. In addition, though using the average characteristic with an ROI is an effective manner of dimensional reduction and noise filtering, ${ }^{10}$ a limitation is its reduced sensitivity to localized anatomic alterations that only affect parts of a predefined structure. ${ }^{11}$ Therefore, we used a voxel-based analysis, which might be considered as an alternative way to explore the imaging makers of presbycusis in the white matter.

\section{MATERIALS AND METHODS \\ Patients}

This study was approved by the Shandong University institutional review board, and each participant provided informed consent. Fifteen patients with mild presbycusis (presbycusis group, 5 men and 10 women; mean age, $63.2 \pm 2.6$ years) who visited the department of otolaryngology of the central hospital of Jinan city were recruited in this study (Table). Hearing loss was defined as a speech-frequency pure-tone average (PTA) of thresholds at $0.5,1$, 2 , and $4 \mathrm{kHz}$ (air conduction) in the better-hearing ear, per the definition of hearing loss adjudicated by the World Health Organization. The PTA value of 25 decibel hearing level (dB HL) was accepted as the normal hearing threshold limit. ${ }^{12}$ Inclusion criteria were mild hearing loss (defined as $25 \mathrm{~dB} H L<$ PTA $\leq 40 \mathrm{~dB}$ $\mathrm{HL}$ in the better-hearing ear) and age $\geq 60$ years. Exclusion criteria were ear diseases that affect hearing thresholds and sensorineural hearing losses other than presbycusis; history of otologic surgery, ototoxic drug therapy, noise exposure, or hearing aid use; asymmetric hearing loss with a difference in air conduction thresholds exceeding $20 \mathrm{~dB}$ in at least 2 frequencies between $0.5,1$, 2 , and $4 \mathrm{KHz}$; conductive hearing loss (the mean air-bone differences at $0.5,1,2$, and $4 \mathrm{KHz})>10 \mathrm{~dB}$ in 1 or both ears; and tinnitus, head trauma, lesions of the facial nerve, disorders of the cervical spine, or neurologic or psychiatric disease. The complete list of exclusion criteria was previously described. ${ }^{13}$

Fourteen age- and sex-matched healthy controls (control group, 6 men and 8 women; mean age, $62.4 \pm 2.0$ years; PTA $\leq 25$ $\mathrm{dB} H \mathrm{HL}$ in the better-hearing ear) were recruited for this study. All controls were in good health and had no history of neurologic or psychiatric disease. Healthy controls were matched for education levels (Table).

None of the subjects were music professionals. All subjects were right-handed according to Li's Handedness Inventory, which was designed for Chinese populations. ${ }^{14,15}$

\section{Assessment of Auditory Function}

An otoscopic examination was performed in all patients to remove cerumen and confirm the presence of an intact tympanic membrane. The hearing abilities of all patients were evaluated by using pure-tone audiometry and tympanometry. The pure-tone audiometry was performed with a Madsen Electronics Midimate 622 Clinical/Diagnostic Audiometer (GN Otometrics, Taastrup, Denmark) coupled with Telephonics TDH-39P headphones (Telephonics, Farmingdale, New York). Bone conduction was measured at $0.25,0.5,1,2$, and $4 \mathrm{KHz}$, and air conduction was measured at $0.125,0.25,0.5,1,2,4$, and $8 \mathrm{KHz}$. Hearing thresholds were detected with a resolution of 5 decibel steps. The PTA values of all patients' ears were calculated. The tympanometry was performed with a Madsen Electronics Zodiac 901 Middle Ear Analyzer (GN Otometrics) to confirm optimal middle-ear conditions.

\section{DTI and Data Processing}

All patients were scanned on a 3T scanner (Achieva TX; Philips Healthcare, Best, the Netherlands) using an 8-channel phasedarray head coil as a receiver. The DTI dataset was acquired with a multisection, single-shot, EPI, spin-echo sequence $(\mathrm{TR} / \mathrm{TE}=$ $6281 / 67 \mathrm{~ms}$; sensitivity encoding factor $=2.5$ ). Sixty-five transverse sections were acquired parallel to the line connecting the anterior commissure to the posterior commissure with no section gap and 2.2-mm nominal isotropic resolution. Diffusion weighting was applied along 32 directions with a b-value of 700 seconds/ $\mathrm{mm}^{2}$. Five minimally weighted images ( $5 \mathrm{~B} 0$ with $b=33$ seconds/ $\mathrm{mm}^{2}$ ) were acquired and averaged on the scanner as part of each DTI dataset.

The raw diffusion-weighted images were first coregistered to the B0 images and corrected for eddy current and patient motion with affine transformation by using Automated Image Registration (http://bishopw.loni.ucla.edu/air5/). ${ }^{16}$ The 6 elements of the diffusion tensor were calculated for each pixel with multivariate linear fitting by using DTIStudio (Johns Hopkins University, Baltimore, Maryland). ${ }^{17}$ After diagonalization, 3 eigenvalues and eigenvectors, as well as FA, MD, DA, and DR, were obtained. A 2-step image transformation was used to warp the individual data to a standard template, JHU-MNI-ss (Johns Hopkins University, 
www.mristudio.org) in the ICBM-152/ICBM-DTI-81 space. First, affine transformation was used to globally adjust the brain position, rotation, and size. Next, a nonlinear transformation with large deformation diffeomorphic metric mapping ${ }^{18}$ was applied to accurately warp the local structures. The dual-contrast large deformation diffeomorphic metric mapping, in which both the B0 image and the FA map were applied simultaneously, was used. These procedures are reciprocal and provide forward (subject to atlas) and backward (atlas to subject) transformation matrices. FA, MD, DA, and DR images then were transformed to template space by using the forward matrix. After the registration, all images were smoothed using statistical parametric mapping by setting the full width at half maximum $=\left[\begin{array}{lll}4 & 4 & 4\end{array}\right]$.

\section{Statistical Analysis}

The 2-tailed Student $t$ test was performed to test for differences in age, education levels, and PTA between patients with presbycusis and healthy controls. The differences in sex were evaluated by a 2-tailed Pearson $\chi^{2}$ test. The threshold of significance was set at $P=.05$. Statistical analyses were performed by using the SPSS version 18.0 software package (IBM, Armonk, New York).

Intergroup statistics were implemented (voxel by voxel) to several DTI indices, including FA, MD, DA, and DR, which had been transformed to the standard space. The results were obtained from SPM8 (http://www.fil.ion.ucl.ac.uk/spm/) by the application of multiple comparisons and the setting of thresholds of $P<.001$ (uncorrected) at voxel level and $P<.05$ (family-wise error-corrected) at cluster level. The minimum cluster size was 100 voxels. The results were visualized by using a Matlab (MathWorks, Natick, Massachusetts) toolbox named xjview (http:// www.alivelearn.net/xjview).

\section{RESULTS}

All patients had a type-A curve on tympanometry, which indicated normal middle-ear function. There was no significant difference in PTA between the left and right ears in the presbycusis group $(P=.49)$ or the control group $(P=.10)$; therefore, the thresholds of both ears were averaged in each group. The PTA was significantly higher in patients with presbycusis than in healthy patients $(P<.001$ [Table] $)$. In the control group, the mean hearing thresholds were $<20 \mathrm{~dB}$ HL at all frequencies; in the presbycusis group, the mean hearing thresholds were $>20 \mathrm{~dB} \mathrm{HL}$ at 1 $\mathrm{kHz}$ and reached $46.8 \mathrm{~dB} \mathrm{HL}$ at $4 \mathrm{kHz}$ and $55.2 \mathrm{~dB} \mathrm{HL}$ at $8 \mathrm{kHz}$ (Fig 1).

The results of voxel-based analyses are shown in Fig 2. The presbycusis group showed increased DA near the right-side Heschl gyrus and increased DR near the right-side Heschl gyrus and the left-side inferior frontal gyrus. The presbycusis group showed decreased FA near the right-side angular gyrus and inferior frontal gyrus and decreased FA near the right-side temporal pole and middle temporal gyrus, which were partly overlapped with acoustic radiation. The presbycusis group also showed increased MD near the right-side Heschl gyrus and the left-side inferior frontal gyrus. The On-line Table specifies the above-mentioned areas distributed in the brain image as voxel clusters with detailed information, including the peak coordinates in the Montreal Neuro-

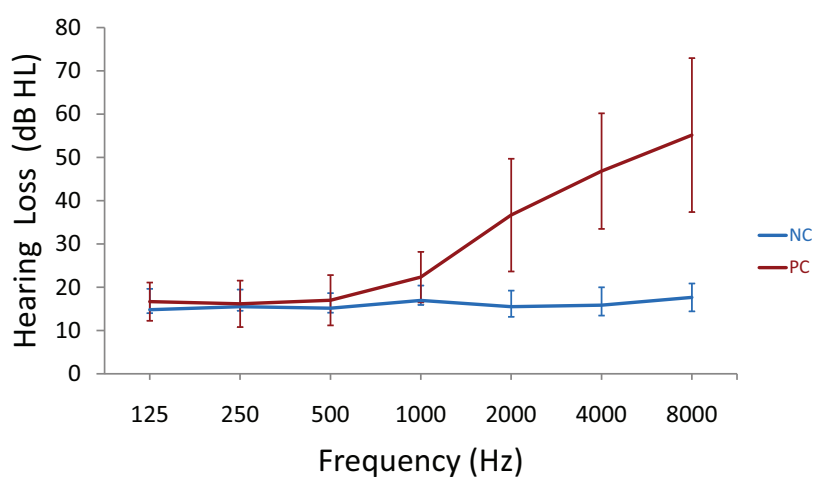

FIG 1. Results of pure-tone audiometry from $125 \mathrm{~Hz}$ to $8000 \mathrm{~Hz}$ (air conduction). Hearing thresholds from both ears are averaged (mean \pm standard deviation) in the presbycusis (PC) and healthy control (NC) groups.

logical Institute space, cluster-level $P$ value, and peak T-value of each cluster.

\section{DISCUSSION}

Our study demonstrated altered DA, DR, FA, and MD near the right-side hearing-related areas of patients with presbycusis compared with age- and sex-matched healthy controls. Increased DR and MD were also observed in a language-related area (Broca area) of patients with presbycusis. To our knowledge, this is the first in vivo demonstration of presbycusis-related changes over the entire brain in a DTI fashion by using a voxel-based analysis.

The scalar measurements of DTI were capable of characterizing various white matter pathologies. Among all of these measurements, FA was sensitive to various microstructural changes ${ }^{19}$; MD was considered the inverse measure of the membrane attenuation and fluid viscosity ${ }^{20}$; DA was considered highly sensitive to axonal degeneration; and DR was sensitive to demyelination. ${ }^{21}$ DTI technique was introduced to study the macroscopic architecture of white matter. However, several animal studies have shown changes of DTI parameters, including MD, DA, and DR, in gray matter areas because of neuron loss. FA did not account for those contributions because the dendrites are only partially coherently organized and the axons are not homogeneously distributed and might be myelinated or unmyelinated in gray matter. ${ }^{22,23}$ When overlaid to the statistical parametric mapping-integrated T1 template, the changes were seen mostly within the subcortical white matter areas with a small portion of voxels overlapped with cortex. Because it is difficult to separate the white matter and gray matter with DTI contrast, the results are elaborated with "gyrus" (which contain both subcortical white matter and cortex) as its units. In our study, increased DA, DR, and MD were found near the right-side Heschl gyrus in patients with presbycusis; decreased FA was also seen in white matter areas that partly overlapped with the right-side acoustic radiation. To further examine the functional representation of the clusters shown in the On-line Table, we overlaid the Brodmann atlas to our results and found that changes overlapped with superior temporal gyrus and angular gyrus were close to Brodmann area 41 , which refers to the auditory cortex. These results could indicate a dysfunctional auditory pathway in presbycusis. This is consistent with previous studies where the DTI-based parameters were changed at the Heschl 

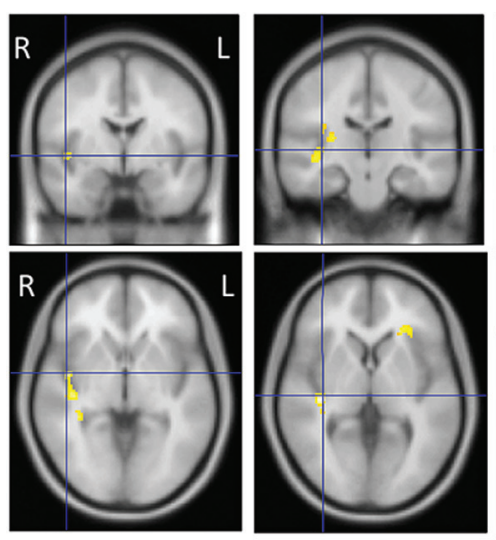

DA Cluster1 DR Cluster1
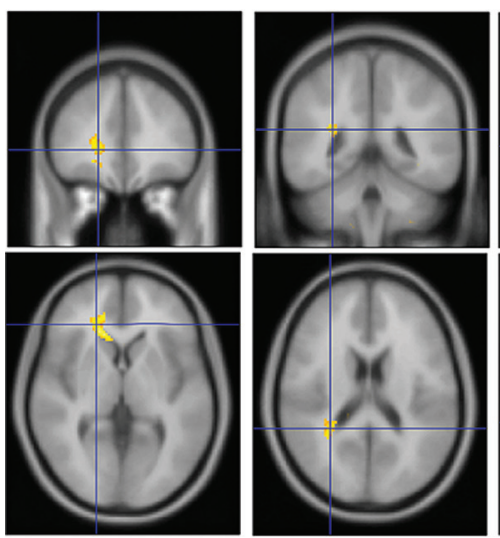

FA Cluster2
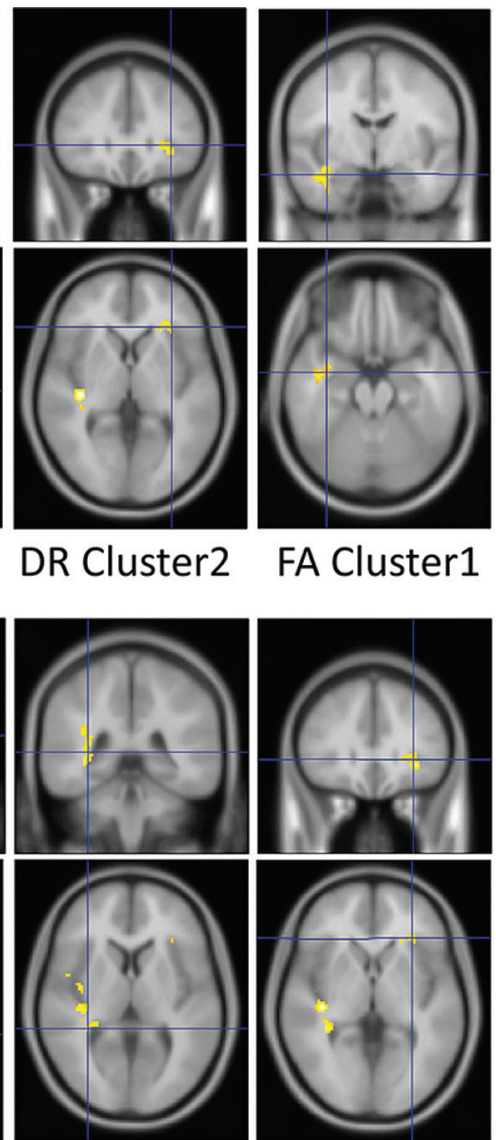

FA Cluster1

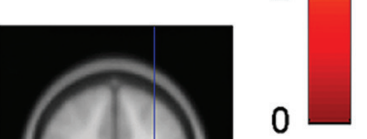

$T$-value

FIG 2. Voxel-based analysis results. The DA, DR, and MD were significantly increased and the FA was significantly decreased in the presbycusis group compared with the healthy control group $(P<.001$ at voxel level, uncorrected; $P<.05$ at cluster level, family-wise errorcorrected). Each cluster contains more than 100 adjacency voxels that show significant variation of those diffusion parameters. For example, DA cluster 1 denotes the first group of voxels that show significant intergroup difference of DA, whereas DR cluster 1 denotes the first group of voxels that show significant intergroup difference of DR. The significant voxels are displayed with their T-values. The ascending T-values are shown from darkness to brightness. Each panel displays a single cluster with its location indicated by a blue section marker.

gyrus, superior temporal gyrus, lateral lemniscus, auditory radiation, and inferior colliculus in patients with sensorineural hearing loss. ${ }^{9,24,25}$ Two possible explanations for the alteration shown in the right-side hearing-related areas are as follows: 1) Presbycusis is most often characterized by a decline in frequency audibility toward high frequencies, which is crucial for pitch perception. One study has demonstrated that the right hemisphere was the dominant hemisphere involved in pitch perception ${ }^{26}$; and 2) Another study found a clear rightward asymmetry in the white matter under the auditory cortex in patients with presbycusis. ${ }^{6}$

To our knowledge, only 1 study has used a DTI-based technique to investigate microstructural changes in the white matter of patients with presbycusis. ${ }^{6}$ In that study, the DA value was increased in the right-side auditory pathway of patients with mild presbycusis compared with young healthy controls. Therefore, the altered integrity of the auditory pathway might be mainly caused by aging. In our study, age-matched healthy patients served as controls, so we determined the altered integrity of the white matter in the right-side Heschl gyrus is mainly driven by hearing loss. However, there were no differences in any measured

plays an important role in auditory processing. ${ }^{33}$

Voxel-based analysis, which is capable of localizing very subtle anatomic changes, suffers from 2 major limitations. First, it searches for the group differences in a hypothesisindependent way. ${ }^{34}$ However, disease-related changes can be distributed in a complex fashion. Therefore, the voxel-based analysis is not sensitive for the detection of small and widely distributed changes in image intensities. Second, voxel-based analysis is sensitive to registration accuracy, which is not reliable enough when based on DTI. This is because DTI does not contain enough structural information compared with T1WI. The registration errors could be reduced by using a more sophisticated approach to further improve accuracy of voxelbased analysis. In addition, DTI is itself limited because it often has to pay a penalty of a higher SNR and higher sensitivity to measurement artifacts such as patient motion and eddy current. ${ }^{35,36}$ Finally, our study was further limited by the small sample size; hence, it should be viewed as a preliminary study and requires confirmation in larger samples.

AJNR Am J Neuroradiol 37:2110-14 Nov 2016 www.ajnr.org 2113 


\section{CONCLUSIONS}

Using a voxel-based analysis, this study revealed presbycusis-related changes of DTI parameters along the auditory pathway and a language-related area. Our findings could be important for exploring more reliable imaging evidence of presbycusis and could complement the ROI-based approach or studies by using different patient populations.

\section{REFERENCES}

1. Schuknecht HF, Gacek MR. Cochlear pathology in presbycusis. Ann Otol Rhinol Laryngol 1993;102(1 Pt 2):1-16 Medline

2. Mazelová J, Popelar J, Syka J. Auditory function in presbycusis: peripheral vs. central changes. Exp Gerontol 2003;38:87-94 CrossRef Medline

3. Snell KB, Frisina DR. Relationships among age-related differences in gap detection and word recognition. J Acoust Soc Am 2000;107: 1615-26 CrossRef Medline

4. Suta D, Rybalko N, Pelánová J, et al. Age-related changes in auditory temporal processing in the rat. Exp Gerontol 2011;46:739-46 CrossRef Medline

5. Ouda L, Druga R, Syka J. Changes in parvalbumin immunoreactivity with aging in the central auditory system of the rat. Expl Gerontol 2008;43:782-89 CrossRef Medline

6. Profant $\mathrm{O}$, Škoch A, Balogová Z, et al. Diffusion tensor imaging and MR morphometry of the central auditory pathway and auditory cortex in aging. Neuroscience 2014;260:87-97 CrossRef Medline

7. Profant $\mathrm{O}$, Tintěra $J$, Balogová $\mathrm{Z}$, et al. Functional changes in the human auditory cortex in ageing. PloS One 2015;10:e0116692 CrossRef Medline

8. Wu CM, Ng SH, Liu TC. Diffusion tensor imaging of the subcortical auditory tract in subjects with long-term unilateral sensorineural hearing loss. Audiol Neurootol 2009;14:248 -53 CrossRef Medline

9. Lin $\mathrm{Y}$, Wang J, Wu C, et al. Diffusion tensor imaging of the auditory pathway in sensorineural hearing loss: changes in radial diffusivity and diffusion anisotropy. J Magn Reson Imaging 2008;28:598-603 CrossRef Medline

10. Miller MI, Faria AV, Oishi K, et al. High-throughput neuro-imaging informatics. Front Neuroinform 2013;7:31 CrossRef Medline

11. Oishi K, Mielke MM, Albert M, et al. DTI analyses and clinical applications in Alzheimer's disease. J Alz Dis 2011;26 Suppl 3:287-96 CrossRef

12. Lin FR, Thorpe R, Gordon-Salant S, et al. Hearing loss prevalence and risk factors among older adults in the United States. J Gerontol A Biol Sci Med Sci 2011;66:582-90 CrossRef Medline

13. Van Eyken E, Van Laer L, Fransen E, et al. KCNQ4: a gene for agerelated hearing impairment? Hum Mutat 2006;27:1007-16 CrossRef Medline

14. Gong G, Jiang T, Zhu C, et al. Side and handedness effects on the cingulum from diffusion tensor imaging. Neuroreport 2005;16: 1701-05 CrossRef Medline

15. Hatta T. Handedness and the brain: a review of brain-imaging techniques. Magn Reson Med Sci 2007;6:99-112 CrossRef Medline

16. Woods RP, Grafton ST, Holmes CJ, et al. Automated image registration: I. General methods and intrasubject, intramodality validation. J Comput Assist Tomogr 1998;22:139-52 CrossRef Medline

17. Jiang H, van Zijl PC, Kim J, et al. DtiStudio: resource program for diffusion tensor computation and fiber bundle tracking. Comput Methods Programs Biomed 2006;81:106-16 CrossRef Medline

18. Ceritoglu C, Oishi K, Li X, et al. Multi-contrast large deformation diffeomorphic metric mapping for diffusion tensor imaging. $\mathrm{Neu}$ roimage 2009;47:618-27 CrossRef Medline

19. Beaulieu C. The basis of anisotropic water diffusion in the nervous system - a technical review. NMR Biomed 2002;15:435-55 CrossRef Medline

20. Alexander AL, Hurley SA, Samsonov AA, et al. Characterization of cerebral white matter properties using quantitative magnetic resonance imaging stains. Brain Connect 2011;1:423-46 CrossRef Medline

21. Alexander AL, Lee JE, Lazar M, et al. Diffusion tensor imaging of the brain. Neurotherapeutics 2007;4:316-29 CrossRef Medline

22. Song SK, Kim JH, Lin SJ, et al. Diffusion tensor imaging detects age-dependent white matter changes in a transgenic mouse model with amyloid deposition. Neurobiol Dis 2004;15:640-47 CrossRef Medline

23. Zerbi V, Kleinnijenhuis M, Fang X, et al. Gray and white matter degeneration revealed by diffusion in an Alzheimer mouse model. Neurobiol Aging 2013;34:1440-50 CrossRef Medline

24. Lutz J, Hemminger F, Stahl R, et al. Evidence of subcortical and cortical aging of the acoustic pathway: a diffusion tensor imaging (DTI) study. Acad Radiol 2007;14:692-700 CrossRef Medline

25. Chang Y, Lee SH, Lee YJ, et al. Auditory neural pathway evaluation on sensorineural hearing loss using diffusion tensor imaging. $\mathrm{Neu}$ roreport 2004;15:1699-703 CrossRef Medline

26. Zatorre RJ, Evans AC, Meyer E, et al. Lateralization of phonetic and pitch discrimination in speech processing. Science 1992;256:846-49 CrossRef Medline

27. Goŕdon-Salant S, Fitzgibbons PJ. Temporal factors and speech recognition performance in young and elderly listeners. J Speech Hear Res 1993;36:1276-85 CrossRef Medline

28. Bao S, Chang EF, Woods J, et al. Temporal plasticity in the primary auditory cortex induced by operant perceptual learning. Nat $\mathrm{Neu}$ rosci 2004;7:974-81 CrossRef Medline

29. Caspary DM, Schatteman TA, Hughes LF. Age-related changes in the inhibitory response properties of dorsal cochlear nucleus output neurons: role of inhibitory inputs. J Neurosci 2005;25:10952-59 CrossRef Medline

30. Burkard RF, Sims D. The human auditory brainstem response to high click rates: aging effects. Am J Audiol 2001;10:53-61 CrossRef Medline

31. Gao F, Wang G, Ma W, et al. Decreased auditory GABA+ concentrations in presbycusis demonstrated by edited magnetic resonance spectroscopy. Neuroimage 2015;106:311-16 CrossRef Medline

32. Manly JJ, Touradji P, Tang MX, et al. Literacy and memory decline among ethnically diverse elders. J Clin Exp Neuropsychol 2003;25: 680-90 CrossRef Medline

33. Bamiou DE, Musiek FE, Luxon LM. The insula (Island of Reil) and its role in auditory processing. Literature review. Brain Res Brain Res Rev 2003;42:143-54 CrossRef Medline

34. Qin YY, Li MW, Zhang S, et al. In vivo quantitative whole-brain diffusion tensor imaging analysis of APP/PS1 transgenic mice using voxel-based and atlas-based methods. Neuroradiology 2013;55: 1027-38 CrossRef Medline

35. Jones DK, Cercignani M. Twenty-five pitfalls in the analysis of diffusion MRI data. NMR Biomed 2010;23:803-20 CrossRef Medline

36. Tournier JD, Mori S, Leemans A. Diffusion tensor imaging and beyond. Magn Reson Med 2011;65:1532-56 CrossRef Medline 